\title{
Magnetic resonance imaging assessment of brainstem distortion associated with a supratentorial mass
}

Suguru Inao, Hiroji Kuchiwaki, Hidemi Kanaiwa, Kenichiro Sugito, Masakazu Banno, Masahiro Furuse

\begin{abstract}
Quantitative measurements of brainstem distortion and neural dysfunction were obtained in 25 cases of chronic subdural haematoma. The horizontal and rotational brainstem displacements were measured on axial and coronal MRI in all patients pre-operatively, and brainstem auditory evoked responses (BAERs) were obtained in 11 cases. Logarithmic relationships were noted on both horizontal and rotational displacements of the
\end{abstract}

brainstem and cerebrum. The type of shift changed in the rostro-caudal direction. In the axial plane, the cerebral hemisphere shifts and rotates, the midbrain shifts laterally with no rotation, and the pons shifts minimally but rotates moderately. In the coronal plane, the marked rotation of the cerebral hemisphere and moderate rotation of the brainstem result in midbrain kinking, suggesting a downward displacement of the midbrain. The prolongation of BAER latencies and cen-
Department of Neurosurgery, Nagoyei University School of Medicine and Department of Neurosurgery, Kohseiren-Kamo Hospital, Japan $S$ Inao

H Kuchiwaki

H Kanaiwa

K Sugita

M Banno

Clinical Imaging

Laboratory,

Department of

Radiology,

Nakatsugawa

Municipal General

Hospital, Japan

M Furuse

Correspondence to: Dr Inao Department of Neurosurgery, Nagoya University Schoo of Medicine, 65

Tsurumai-cho, Showa-ku, Nagoya 466, Japan

Received 11 February 1992 and in revised form 7 April 1992.

Accepted 19 June 1992
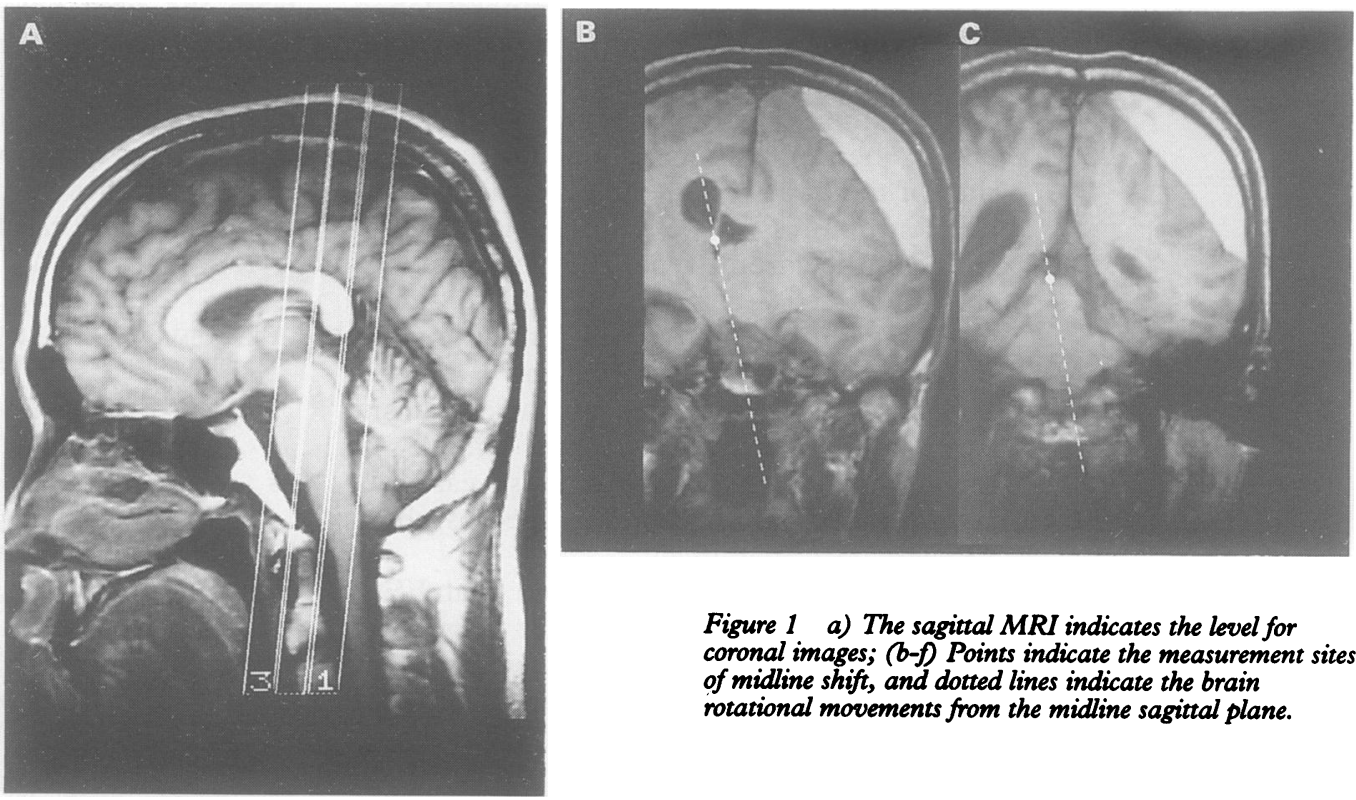

Figure 1 a) The sagittal MRI indicates the level for coronal images; (b-f) Points indicate the measurement sites of midline shifi, and dotted lines indicate the brain rotational movements from the midline sagittal plane.

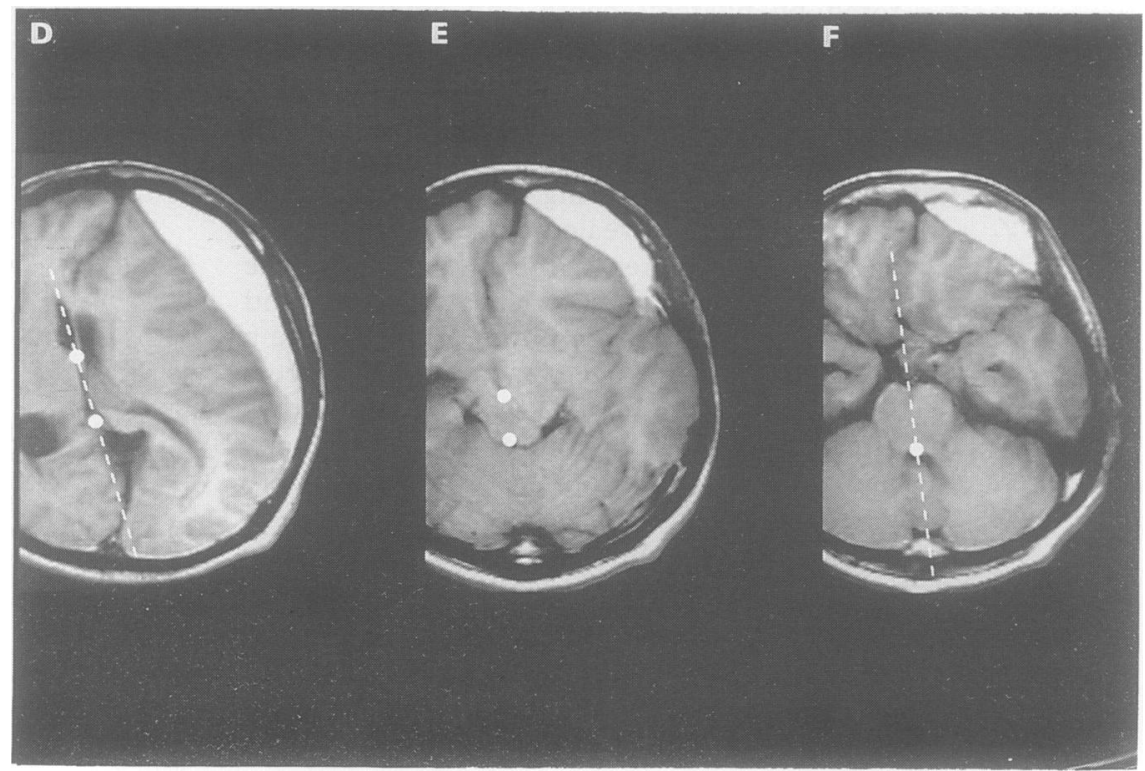


tral conduction times correlated with septum shift. The results of peak-V latency indicated that brainstem rotation in the coronal plane reflects upper brainstem dysfunction most closely. This study presents objective measurements of brainstem displacement shown on MRI, and clarifies the relationships between anatomical and physiological changes in the brainstem that are associated with supratentorial lesions.

(F Neurol Neurosurg Psychiatry 1993;56:280-285)

Recent developments in neuroradiological imaging techniques allow better understanding of the correlation between neural dysfunction and anatomical changes. CT demonstrated horizontal displacement of the brainstem associated with an acute unilateral
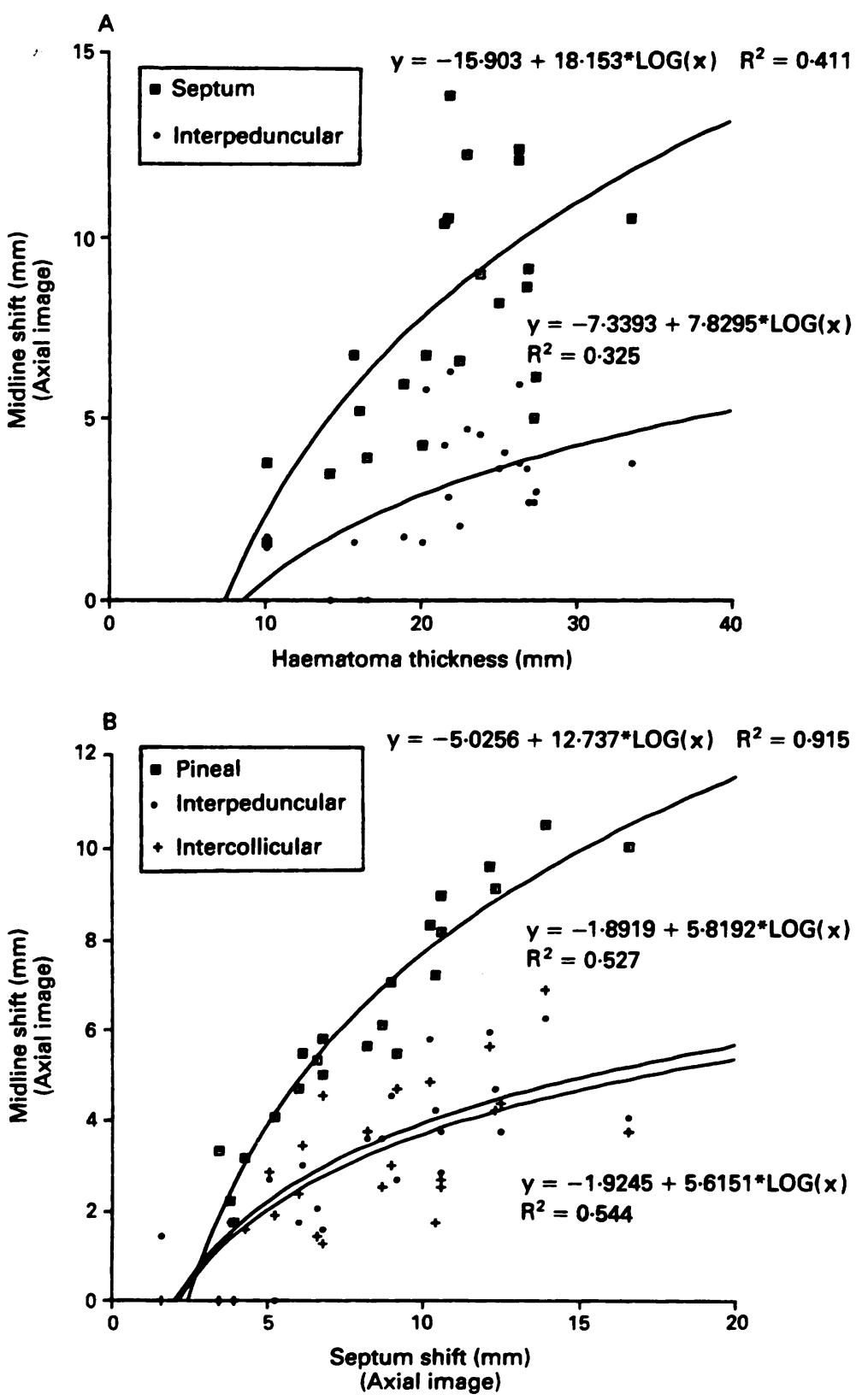

Figure 2 a) Logarithmic relationships were noted in haematoma thickness versus midline shift of septum pellucidum and interpeduncular fossa; b) Shifts of the pineal, interpeduncular fossa and intercollicular sulcus were also logarithmically related to septum shift (see text).
Table 1 Summary of MRI data of haematoma thickness, midline shifts and rotational angles

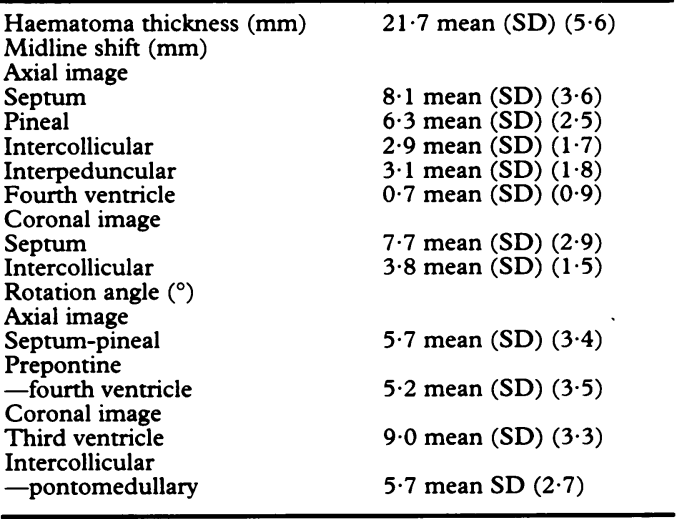

cerebral mass relates closely to depressed consciousness. ${ }^{12}$ Although brainstem distortion itself is implicated in the mechanism of depressed consciousness, transtentorial herniation does not consistently correlate with the clinical status of the patient. ${ }^{13}$ Few studies, however, have attempted to quantify brainstem distortion or derive relationships between cerebral shift and brainstem distortion in patients with supratentorial spaceoccupying lesions. Using MRI, we measured various parameters of brainstem distortion associated with cerebral hemispheric displacement secondary to a unilateral supratentorial mass. Horizontal and rotational displacements of the cerebral hemisphere and brainstem were measured in the axial and coronal planes. Brainstem auditory evoked responses (BAERs) were obtained to derive parameters of brainstem function. Various relationships were analysed to derive a mechanism for brainstem dysfunction in patients with a supratentorial mass.

\section{Material and methods}

Twenty five patients with unilateral chronic subdural haematoma [age range 39-87, mean (SD) 66.4 (3.5) years] were analysed for this study. Patients had symptoms of hemiparesis or headache or a change in mental status, or a combination of the above for more than a month. A history of trauma was elicited in $80 \%(20 / 25)$ of the cases. Patients with bilateral lesions or concomitant other cerebral pathology (normal pressure hydrocephalus, cerebral infarction, or other types of haematoma) were excluded.

Both axial and coronal T1-weighted MRIs were obtained in all patients pre-operatively using a Shimazu SMT $150,1.5$ tesla MRI system (TR $500 \mathrm{~ms}$, TE $26 \mathrm{~ms}, 10 \mathrm{~mm}$ thickness and $1 \mathrm{~mm}$ gap, 256 by 256 pixels). Axial images were taken along the orbitomeatal-line and coronal images were taken along the line through the quadrigeminal plate and pontomedullary junction (fig 1a). The line for the coronal image used in this paper is approximately perpendicular to the orbitomeatalline. In our hospital, we routinely obtain coronal MRIs along this line through the 
A

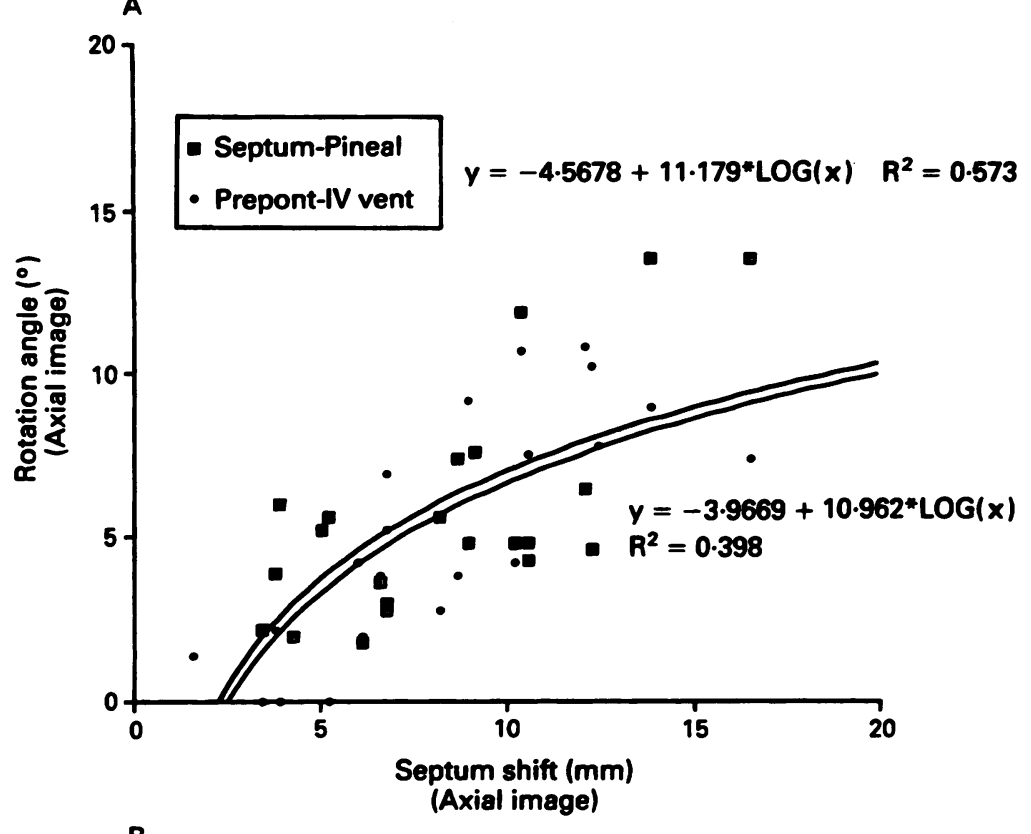

B

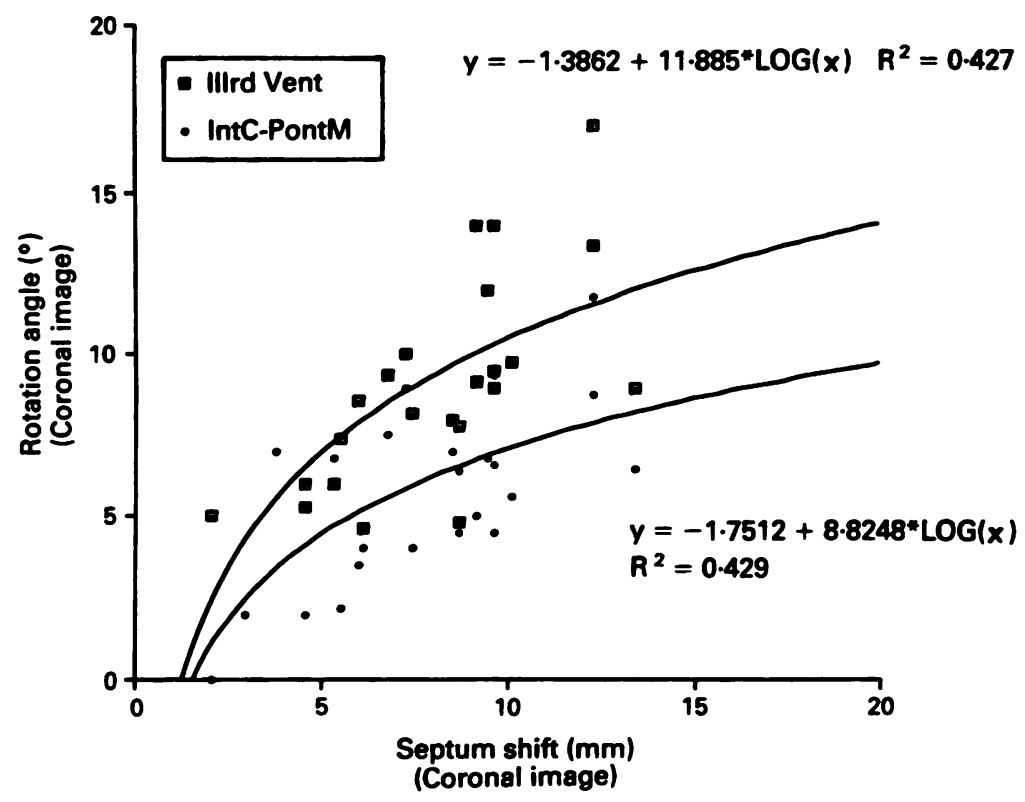

Figure 3 a) Brain rotational movements in the axial plane changed logarithmically; b) In the coronal plane, cerebral and brainstem rotations were related to septum shift logarithmically. The difference in magnitude of rotation between cerebrum and brainstem at higher level of cerebral shfts could result in midbrain kinking. Prepont-IVvent:

Prepontine-Forth ventricle; IntC-PontM: Intercollicular-Pontomedullary.

quadrigeminal plate and pontomedullary junction. The maximum haematoma thickness was measured on the coronal image (fig 1b-c). The shift from the midline sagittal plane of the septum pellucidum (between the anterior horns of the lateral ventricles), pineal body, vertical intercollicular sulcus (or aqueduct), interpeduncular fossa, and the floor of the fourth ventricle were measured on axial images (fig 1d-f). The shifts of the septum pellucidum and intercollicular sulcus at the level of superior colliculus were also measured on coronal images (Fig $1 \mathrm{~b}-\mathrm{c}$ ). The values were converted to true dimensions by means of the gradicule on the radiograph. Rotational displacements were measured as angles between the midline sagittal plane and the following lines: on axial images, a line connecting the septum pellucidum and pineal body, and another line connecting the midpoint of the prepontine cistern and the floor of the fourth ventricle; on coronal images, a line through the plane of the third ventricle (the incline of the third ventricle), and another line connecting the intercollicular sulcus and midpoint of the pontomedullary junction. The angles on coronal views provided measures of downward displacement of the brainstem.

In 11 cases, brainstem auditory evoked responses (BAERs) were performed pre-operatively. The latency of each peak (from I to V) and central conduction time were compared with normal subjects. Statistical analysis was performed using one sample of normal tests for the comparisons between normal subjects and the patient group.

\section{Results}

MRI FINDINGS

Haematoma thickness and midline shift

The average values of haematoma thickness and measurements of midline shift are summarised in table 1 . There were no significant differences between septum and intercollicular shift on both axial and coronal images. The results showed that the septum is most shifted and the fourth ventricle shift is least displaced. Logarithmic relationships were noted between both septum (cerebral) and interpeduncular fossa (brainstem) shifts, and haematoma thickness with fair correlations (septum $r=0.64$, interpeduncular $r=0.57$ ) (fig 2a). Shifts of the pineal body and other brainstem structures were also logarithmically related to septum shift, but with better regression coefficients (pineal $r=0.96$, interpeduncular $r=0.73$, and intercollicular $r=0.74$ ) (fig 2b). Brainstem displacement was not evident until the septum (cerebral) shift was about 3-5 mm. With septum shifts between $5 \mathrm{~mm}$

Table 2 Brainstem auditory evoked response (BAER) data

\begin{tabular}{|c|c|c|c|c|c|c|c|}
\hline & \multicolumn{5}{|c|}{ Peak latency } & \multicolumn{2}{|c|}{ Central conduction time } \\
\hline & I & II & III & IV & $\mathbf{v}$ & $\mathrm{I}-\mathrm{V}$ & III-V \\
\hline $\begin{array}{l}\text { All Patients } \\
(n=11) \\
\text { Septal Shift } \\
\text { 8mm }>(n=5) \\
\text { Septal Shift } \\
\text { 8mm }<(n=6) \\
\text { Control }\end{array}$ & $\begin{array}{l}1.65 \\
(0 \cdot 10) \\
1 \cdot 68 \\
(0 \cdot 13) \\
1 \cdot 62 \\
(0.08) \\
1 \cdot 63 \\
(0.07)\end{array}$ & $\begin{array}{l}2 \cdot 84^{\star \star} \\
(0 \cdot 21) \\
2 \cdot 91^{\star \star} \\
(0 \cdot 27) \\
2 \cdot 76 \\
(0 \cdot 09) \\
2 \cdot 72 \\
(0 \cdot 10)\end{array}$ & $\begin{array}{l}3.94^{\star \star} \\
(0 \cdot 14) \\
4 \cdot 02^{\star \star} \\
(0 \cdot 13) \\
3 \cdot 87 \\
(0 \cdot 11) \\
3 \cdot 78 \\
(0 \cdot 10)\end{array}$ & $\begin{array}{l}5 \cdot 15^{\star \star} \\
(0 \cdot 14) \\
5 \cdot 22^{\star \star} \\
(0 \cdot 18) \\
5 \cdot 11 \\
(0 \cdot 10) \\
4 \cdot 96 \\
(0 \cdot 22)\end{array}$ & $\begin{array}{l}5 \cdot 96^{\star \star} \\
(0 \cdot 14) \\
6 \cdot 04^{\star \star} \\
(0 \cdot 13) \\
5 \cdot 88 \\
(0 \cdot 11) \\
5 \cdot 80 \\
(0 \cdot 20)\end{array}$ & $\begin{array}{l}4 \cdot 29 \star \star \\
0 \cdot 15) \\
4 \cdot 37^{\star \star} \\
(0 \cdot 10) \\
4 \cdot 23 \\
(0 \cdot 16) \\
4 \cdot 12 \\
(0 \cdot 22)\end{array}$ & $\begin{array}{l}2 \cdot 00^{\star \star} \\
(0 \cdot 11) \\
2 \cdot 02^{\star \star} \\
(0 \cdot 10) \\
1 \cdot 98^{\star \star} \\
(0 \cdot 13) \\
1 \cdot 90 \\
(0 \cdot 10)\end{array}$ \\
\hline
\end{tabular}

( ): SD; ${ }^{\star} p<0.05 ;{ }^{\star \star} p<0.01$. 
A

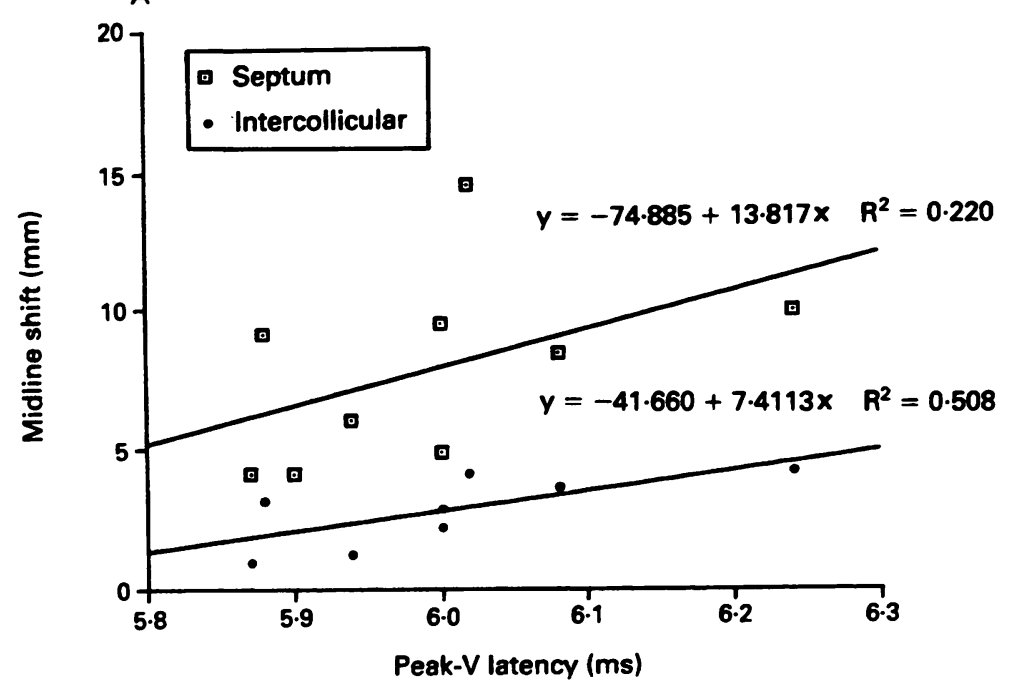

B

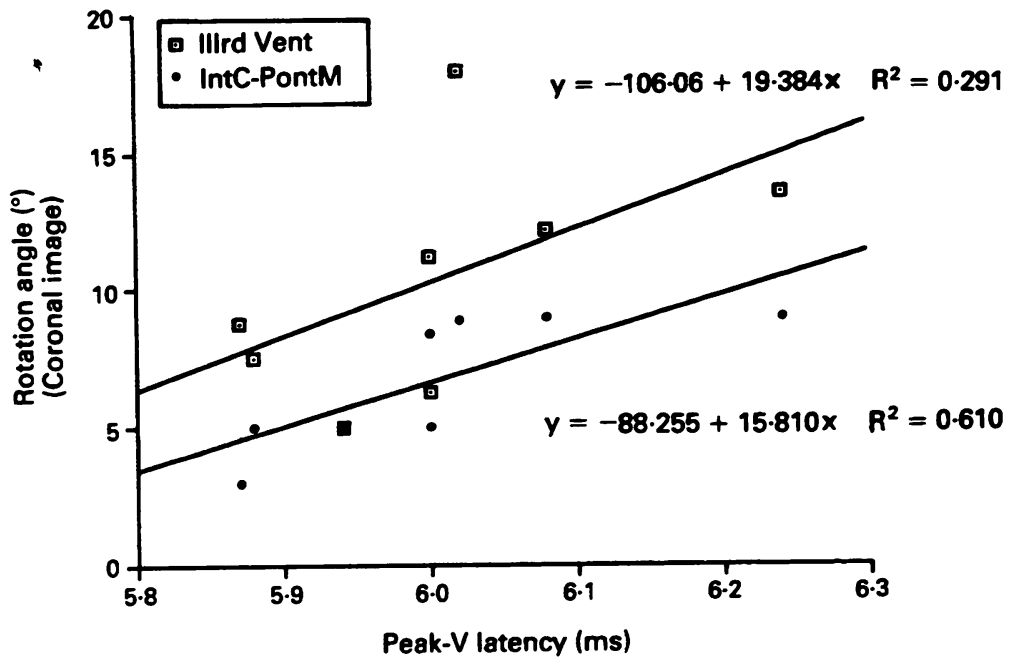

Figure 4 Peak-V latency appears to correlate well with horizontal shifts of the intercollicular sulcus (a) and with rotations of the intercollicular-pontomedullary line (b). IIIrd vent: Third ventricle; IntC-PontM: Intercollicular-Pontomedullary.

and $10 \mathrm{~mm}$, a marked shift of the brainstem was noted, reaching a plateau in displacement even beyond $10 \mathrm{~mm}$ of septum shift. The results also show that shifts of the midbrain are about $1 / 2$ of those of the pineal body. Furthermore, both interpeduncular fossa and intercollicular sulcus were displaced equally possibly indicating that the midbrain moves parallel to the sagittal plane without rotational movement in the axial plane.

\section{Brain tissue rotation}

Angles of brain rotational displacement are summarised in table 1 . In the axial plane, rotations of septum-pineal (central cerebrum) line and prepontine-fourth ventricle (brainstem) line are logarithmically related to septum shift (fig 3a). Although the rotational movement of the brainstem changed by the same magnitude as the cerebrum, its axis of rotation is different. The axis of cerebral rotation is displaced from the mid-sagittal plane, while the axis of pontine rotation was centred around the almost fixed position of the fourth ventricle. In the coronal plane, rotational movements of the third ventricle (cerebrum) line and intercollicular sulcus-pontomedurally junction (brainstem) line are shown to be logarithmically related to septum shift (fig $3 \mathrm{~b})$. The difference in magnitude of rotation in this plane between the cerebrum and brainstem at higher levels of cerebral shifts results in midbrain kinking.

\section{CLINICAL DATA}

Twelve patients [mean (SD) age $63.5(17 \cdot 2)$ ] had only headache and/or vomiting without other neurological deficits and a septum shift of $7.16(4.26) \mathrm{mm}$. The other thirteen patients [mean (SD) age $71.8(9.4)$ ] had hemiparesis and/or change in mental status or consciousness disturbance (drowsiness), and a septum shift of $9.04(2.98) \mathrm{mm}$. These values of septum shift are not statistically different using Student's $t$ test.

BAER data are summarised in table 2. The patients were tentatively divided into two groups based on a septum shift of greater than or less than $8 \mathrm{~mm}$ (mean value of septum shift for entire group) to determine the effect of supratentorial shift on the brainstem function. Latencies of the second through fifth peaks of BAERs, labelled sequentially from I to V, appeared significantly prolonged when averaged from all patients $(n=11)$. However, in patients with less than $8 \mathrm{~mm}$ of septum shift $(n=6)$, average peak latencies were not significantly changed from controls. Central conduction times measured as the interpeak latencies between $I$ and $V$, and between III and V were also similarly prolonged in all patients. Here, however, in patients with less than $8 \mathrm{~mm}$ septum shift, the average III to $\mathrm{V}$ interpeak latency was prolonged but the average $\mathrm{I}$ to $\mathrm{V}$ interpeak latency was not significantly changed. Prolongation of peak- $V$ latency appears to correlate best with angulation of the intercollicular-pontomedullary line (coronal brainstem rotation) $(r=0.78)$. It does not seem to relate as well to septum shift $(r=0.47)$, or third ventricular angulation ( $r=0.54$ ) (fig 4).

\section{Discussion}

MRI is used to derive various parameters of brainstem and cerebral displacements in patients with a supratentorial mass. Brainstem deformity correlates well with BAER data.

\section{MRI assessment of brainstem deformity}

Many clinico-pathological studies ${ }^{45}$ indicate that brainstem distortion associated with a supratentorial mass is one of the most important factors influencing neurological deterioration with or without transtentorial herniation. Brainstem deformity was classified into two $^{67}$ or three types ${ }^{8}$ in pathological materials; lateral (or horizontal) displacement, downward (or caudal) displacement, and rotation in the axial plane. Sunderland reported that these types of displacements changed as transtentorial herniation progressed. CT scans have shown that the brainstem begins to rotate and shift in the initial 


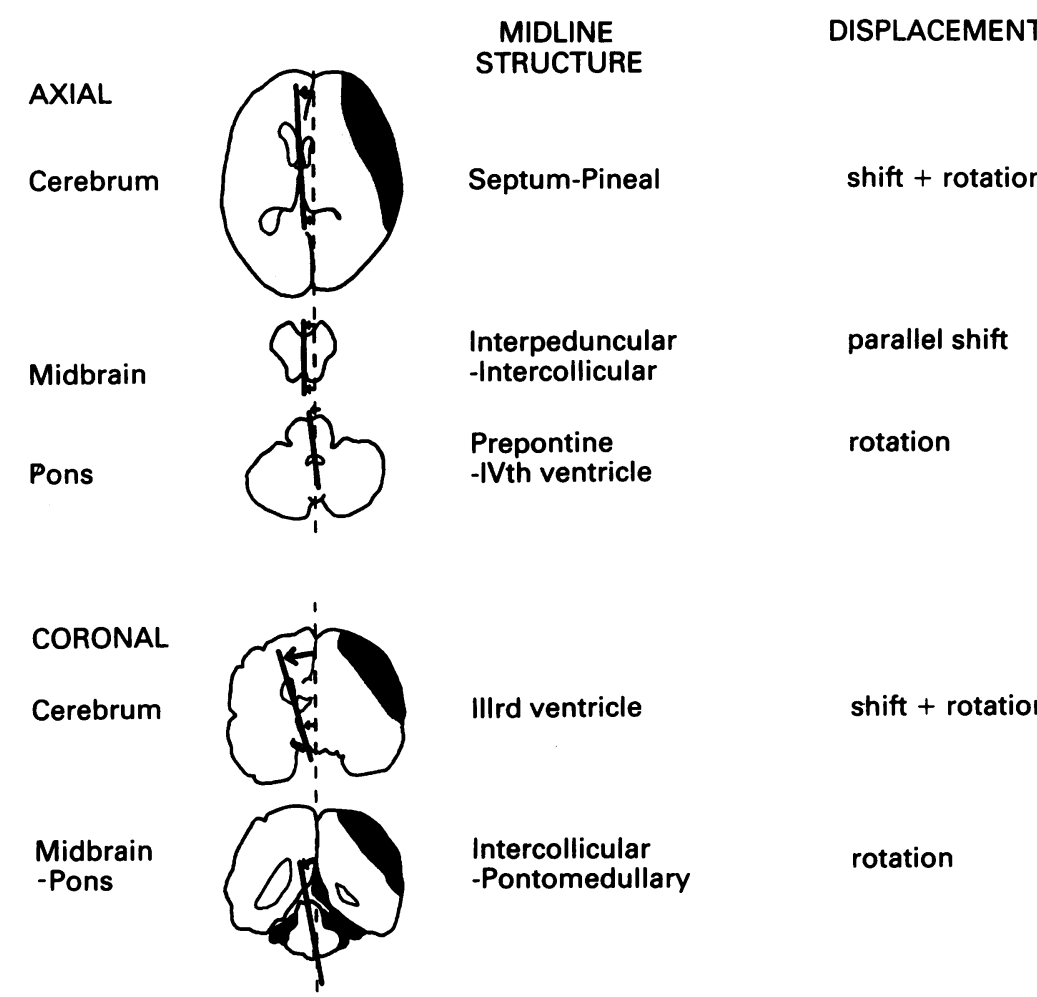

Figure 5 The type of shift changes in the rostro-caudal direction. In the axial plane, the cerebral hemisphere shifts and rotates, the midbrain shifts laterally with no rotation, and the pons shifts minimally but rotates moderately. In the coronal plane, the marked rotation of the cerebral hemisphere and moderate rotation of the brainstem result in midbrain kinking. of the supratentorial mass. Our data was obtained from patients with a chronic subdural haematoma in a relatively uniform location so that brainstem displacement was primarily affected by the size of the haematoma. The extent of brain atrophy is also an important factor since it allows for a larger mass before brainstem displacement occurs. ${ }^{12}$ This partly accounts for the relatively poor correlation between haematoma size and septum shift in our patients. For this reason brainstem distortion was compared with septum shift rather than haematoma size. With older patients, brainstem deformity might be further modified by brainstem atrophy itself. Howell ${ }^{6}$ reported that the white matter of the medulla, basis pontis, and cerebral peduncles resists compression better than the grey matter of the diencephalon and tegmentum of the midbrain and pons, resulting in brainstem buckling; the posterior half of the midbrain and pons shortening more than the anterior half. Our observations confirm that kinking does occur in the midbrain when a supratentorial mass is present. However, this was noted in the coronal plane and measurements in the sagittal plane were not carried out.

Several authors report that it is difficult to identify the downward shift of the brainstem by CT. ${ }^{1210}$ Measurements of caudal displacement of pineal calcification from the level of the opposite lateral ventricular choroid calcification have failed to reveal significant findings due to poor CT resolution. Using coronal images on MRI, downward shift of the brainstem was identified but difficult to measure directly due to the lack of a standard horizontal line. As a measure of this displacement we used the angle between the displaced neuro-axis and the vertical midline in the coronal plane. The coronal image presents the best view to observe midbrain deformity.

\section{Correlation between BAER and brainstem deformity}

For a more objective assessment, we obtained BAERs to compare these with brainstem deformity. Tsubokawa et $a^{13}$ reported that in acute severe head injury patients' BAERs provide more reliable information about brainstem function than the neurological signs or CT findings. Our results show that brainstem deformity measured by MRI correlates well with brainstem dysfunction as assessed by BAERs. Despite the small number of cases, our findings of prolongation of BAER latencies and central conduction times correlated with septum shift. All peak latencies, except peak-I, and central conduction times were prolonged in the severe septum shift group indicating midbrain as well as pontine involvement; while in the mild shift group prolongation of only the III-V central conduction time suggests only midbrain dysfunction. Experimental data has shown that peak-V latency may be a sensitive index of caudal displacement of the upper brainstem. ${ }^{14}$ In our study, the results of peak- $\mathrm{V}$ latency 
indicated that brainstem rotation in the coronal plane reflects upper brainstem (midbrain) dysfunction most closely, which is in agreement with experimental data..$^{14}$

This study presents objective measurements of brainstem displacement as noted on MRI, and clarifies the relationships between anatomical and physiological changes in the brainstem that are associated with supratentorial lesions.

We are grateful to Drs. Sunil J Patel, Kazuhiko Andoh, Taijiro Hirano, Kazuo Yoshida, and Akira Izawa for valuable advice and help with manuscript preparation.

1 Ropper AH. Lateral displacement of the brain and level of consciousness in patients with an acme hemispheral mass. N Engl f Med 1986;314:953-8.

2 Ross DA, Olsen WL, Ross AM, et al. Brain shift, level of consciousness, and restoration of consciousness in patients with acute intracranial hematoma. $₹$ Neurosurg 1989;71:498-502.

3 Fisher CM. Acute brain herniation-a revised concept. Semin Neurol 1984;4:.

4 Munro D, Sisson WR Jr. Hernia through the incisura of the tentorium cerebelli in connection with craniocerethe tentorium cerebelli in connection with cral
bral trauma. N Engl $\mathcal{F}$ Med 1952;247:699-708.
5 Schwarz GA, Rosner AA. Displacement and herniation of the hippocampal gyrus through the incisura tentorii: a clinicopathologic study. Arch Neurol Psychiatry 1941;46: 297-321.

6 Howell DA. Upper brain-stem compression and foraminal impaction with intracranial space-occupying lesions and impaction with intracranial space-

7 Johnson RT, Yates. PO Brain stem haemorrhages in expanding supratentorial conditions. Acta radiol 1956;

8 Sunderland $S$. The tentorial notch and complications produced by herniations of the brain through that aperture. Br $\mathcal{F}$ Surg 1958;45:422-38.

9 Osborn AG. Diagnosis of descending transtentorial herniation by cranial computed tomography. Radiology 1977 123:93-96.

10 Storvring J. Descending tentorial herniation: Findings on computed tomography. Neuroradiology 1977;14: 101-105.

11 Andrews BT, Chiles BW, Olsen WL, et al. The effect of intracerebral hematoma location on the risk of brainstem compression and on clinical outcome. $\mathcal{f}$ Neurosurg 1988;69:518-22.

12 Fogelholm $R$, Heiskanen $O$, Waltimo $O$. Chronic subdural hematoma in adults. Influence of patient's age on dural hematoma in adults. Influence of patient's age on
symptoms, signs, and thickness of hematoma. $\mathcal{F}$ symptoms, signs, and
Neurosurg 1975;42:43-46.

13 Tsubokawa $\mathrm{T}$, Nishimoto $\mathrm{H}$, Yamamoto $\mathrm{T}$, et al. Assessment of brainstem damage by the auditory brainstem response in acute severe head injury. $\mathcal{f}$ Neurol Neurosurg Psychiatry 1980;43:1005-11.

14 Nagao S, Roccaforte P, Moody R. Acute intracranial hypertension and auditory brain-stem responses. $\mathrm{Pt} 2$ : The effects of brain-stem movement on the auditory brain-stem responses due to transtentorial herniation. $f$ Neurosurg 1979;51:846-51. 